\title{
Surgical anatomy of supratentorial midline lesions
}

\author{
M. Gazi Yaşargil, M.D., UĞUr Türe, M.D., and Dianne C. H. Yaşargil, R.N. \\ Departments of Neurosurgery, University of Arkansas for Medical Sciences, Little Rock, Arkansas; \\ and Ondokuz Mayis University School of Medicine, Samsun, Turkey
}

\begin{abstract}
Object. In this paper the authors correlate the surgical aspects of deep median and paramedian supratentorial lesions with the connective fiber systems of the white matter of the brain.

Methods. The cerebral hemispheres of 10 cadaveric brains were dissected in a mediolateral direction by using the fiber dissection technique, corresponding to the surgical approach.

Conclusions. This study illuminates the delicacy of the intertwined and stratified fiber laminae of the white matter, and establishes that these structures can be preserved at surgical exploration in patients.
\end{abstract}

\section{KEY WORDS - cingulum - fiber dissection technique • interhemispheric approach • limbic system}

Falcine meningiomas; AVMs; cavernomas; intrinsic tumors of the medial frontal, parietal, and occipital lobes, cingulate gyrus, corpus callosum, parasplenial region, thalamus, and hypothalamus; and also lesions in the third and lateral ventricles can now be successfully explored and removed via an anterior, middle, or posterior interhemispheric approach, or via a supracerebellar-transtentorial approach, without causing injury. ${ }^{1-7,9,11-17,20-27}$ Comprehensive descriptions of the related surgical anatomy and the specific surgical strategy, tactics, and techniques can be found in earlier publications..$^{22-25}$

\section{DISSECTION}

\section{Preparation of Specimens}

Ten previously frozen, formalin-fixed human brains were dissected with the aid of an operating microscope by using the fiber dissection technique, beginning at the medial aspect and proceeding to the lateral aspect in a step-bystep fashion. ${ }^{8,10,18,19}$ The brains were obtained as fresh autopsy specimens and preserved in $10 \%$ formalin solution for at least 2 months. Following the period of fixation, the pia mater, arachnoid mater, and vessels of each specimen were carefully removed under the operating microscope. The brains were washed with running water for several hours to eliminate the formalin, drained, and then refrigerated for 1 week at a temperature of -10 to $-15^{\circ} \mathrm{C}$. Before we commenced dissection, the brains were immersed in water and allowed to thaw.

\section{Surgical Tools}

The dissection was performed with the aid of an operating microscope, using 4 to $10 \times$ magnification. During the

\footnotetext{
Abbreviations used in this paper: $\mathrm{AVM}=$ arteriovenous malformation; $\mathrm{CNS}=$ central nervous system $; \mathrm{CT}=$ computerized tomography; $\mathrm{MR}=$ magnetic resonance.
}

freezing process, formalin ice crystals form between the nerve fibers, expanding and thus separating the fibers. This freezing process facilitates dissection of the fine fiber bundles in particular. Our primary dissection tools were thin, soft, wooden spatulas with various sized tips $(1,2,4$, and 6 $\mathrm{mm}$ ) and a surgical suction system. Soft wooden spatulas were used to peel away the fiber bundles along anatomical planes, and high-power suction aided the process.

\section{Mediolateral Dissection of the White Matter}

Dissection of the medial aspect of the cerebral hemisphere proceeds as follows (Fig. 1A). The midbrain is severed to achieve adequate visualization of the mediobasal temporal region. Dissection begins at the anterior cingulate sulcus and extends posteriorly until the cingulate cortex has been removed. Dissection proceeds along the callosal sulcus and extends from the subcallosal area to the hippocampal sulcus posteriorly. The indusium griseum and lateral and medial longitudinal striae become visible within the callosal sulcus. The indusium griseum is an extension of the hippocampal formation, a fine layer covering the corpus callosum. The indusium griseum extends anteroinferiorly to the paraterminal gyrus, which merges into the diagonal band of Broca, located in the anterior perforated substance. The cingulum is demonstrated around the corpus callosum, and extends as far as the subcallosal area. The connections of the cingulum to the frontal, precentral, postcentral, and precuneal areas are illustrated. The arcuate or U fibers underlying the medial frontoparietal cortex are also displayed. Removing the cortex of the parahippocampal gyrus exposes the inferior arm of the cingulum. The uncus and uncal sulcus are identified, the uncal sulcus being an extension of the hippocampal sulcus. Retracting the cingulum beneath the splenium exposes the tail of the hippocampus and the subsplenial gyrus (Fig. 1B).

After cutting through the medial portion of the corpus 


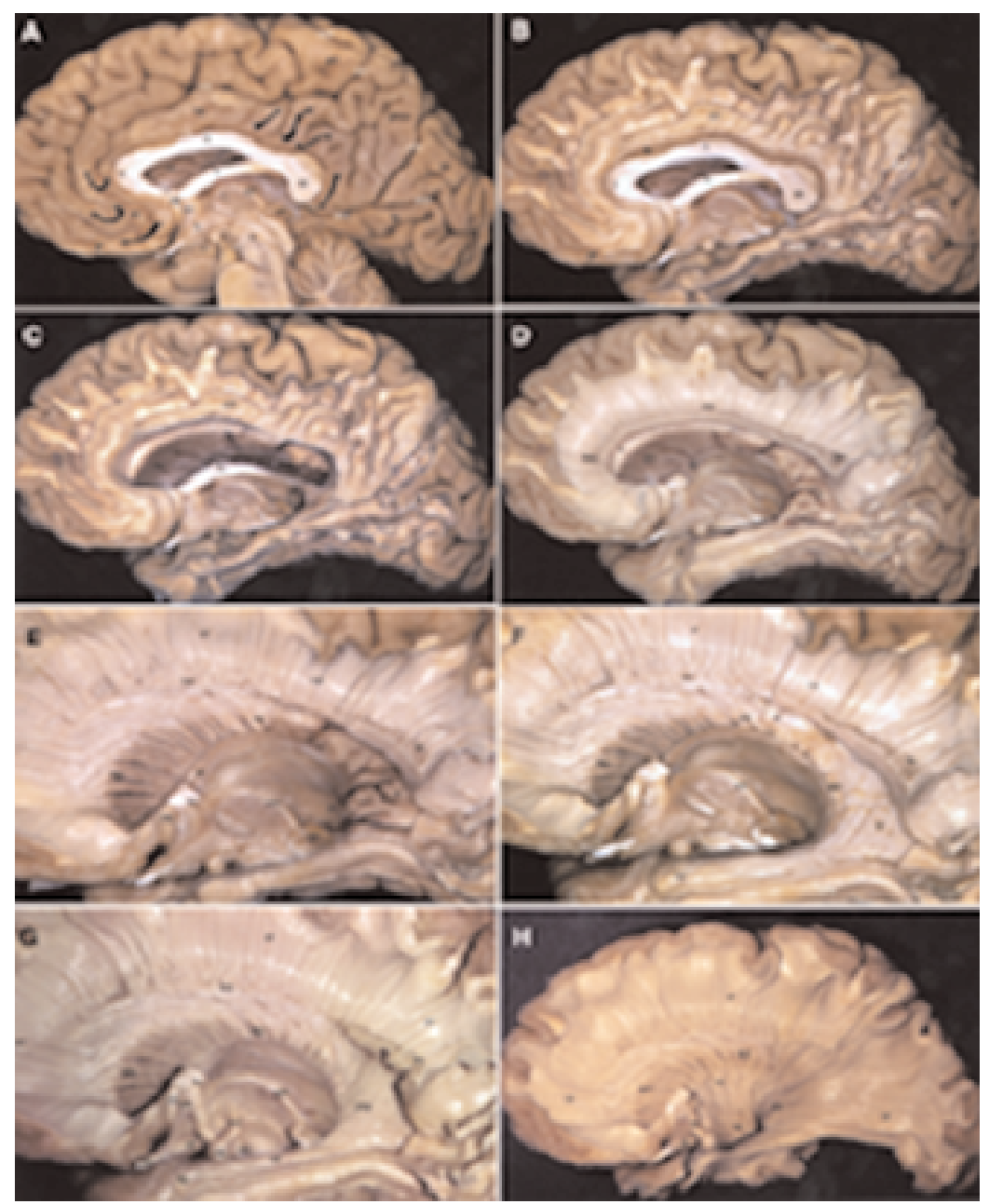

Fig. 1. Photographs of cadaveric brains showing serial dissections of the medial aspect of the left cerebral hemisphere. A: Medial aspect of the right cerebral hemisphere. Connective arms (arrows) link the cingulate gyrus to gyrus rectus, to anterior, middle, and posterior portions of the medial frontal gyrus, and to the precuneus, and the structure continues as a parahippocampal gyrus. B: The midbrain is removed to achieve sufficient visualization of the mediobasal temporal region. Dissecting the cortex of the cingulate and parahippocampal gyrus demonstrates the whole length of the cingulum and its connections. C: After cutting the medial portion of the corpus callosum, the caudate nucleus in the lateral wall of the lateral ventricle is demonstrated. D: Removal of the choroid plexus, cingulum, and mediobasal temporal region, dissection of the radiating fibers of the corpus callosum, and partial removal of the fornix and amygdala further reveals the stria terminalis, the thalamus, and the hypothalamus, which are covered by the transparent ependyma. E: Partial removal of the ependyma and the caudate nucleus in the lateral ventricle, and dissecting away the subcallosal stratum and the anterior portion of the radiation of the corpus callosum demonstrates cortical extensions of the anterior and superior thalamic peduncles as well as the corona radiata and the intersection of the corpus callosum with the corona radiata. F: Following total removal of the ependyma and caudate nucleus, the tapetum of the corpus callosum and the posterior and inferior thalamic peduncles are demonstrated. G: The tapetum, the stria terminalis, and the amygdala have been dissected away. The thalamus and fibers of the anterior, superior, posterior, and inferior thalamic peduncles as well as the optic radiation on the roof of the temporal horn are demonstrated. Dissecting away the hypothalamus and thalamus demonstrated the column of the fornix and the mammillothalamic tract. $\mathrm{H}$ : Removal of the hypothalamus and thalamus with anterior, superior, posterior, and inferior peduncles (the mammillary body and column of the fornix are preserved) demonstrates the lateral portion of the corona radiata and internal capsule. $\mathrm{A}=$ amygdala; $\mathrm{ac}=$ anterior commissure; acs = anterior calcarine sulcus; af $=$ arcuate fibers; atp = anterior thalamic peduncle (internal capsule); $\mathrm{b}=$ body of corpus callosum; $\mathrm{bf}=$ body of fornix; $c \mathrm{c}=$ corpus callosum; ces = central sulcus; $\mathrm{cf}=\mathrm{column}$ of fornix; $\mathrm{cg}=\mathrm{cingulate}$ gyrus; chp = choroid plexus; cin = cingulum; cis = cingulate sulcus; $\mathrm{cn}=$ caudate nucleus; cols = collateral sulcus; $\mathrm{cp}=$ cerebral peduncle; $\mathrm{cr}=$ corona radiata; $\mathrm{cs}=$ callosal sulcus; $\mathrm{cu}=$ cuneus; $\mathrm{e}=$ ependyma; $\mathrm{fg}=$ fusiform gyrus; $\mathrm{fm}=$ forceps major (radiation of corpus callosum); $\mathrm{fmi}=$ forceps minor (radiation of corpus callosum); fo = fornix; $\mathrm{g}=$ genu of corpus callosum; $\mathrm{gr}=$ gyrus rectus; $\mathrm{h}=\mathrm{hypothalamus}$; ic $=$ internal capsule; icc $=$ intersection of corpus callosum with corona radiata; ic $1=$ frontopontine tract (internal capsule); ic $2=$ pyramidal tract (internal capsule); ic3 = occipitopontine tract (internal capsule); ic4 = temporopontine tract (internal capsule); ig = indusium griseum; ist = isthmus cinguli; itp = inferior thalamic peduncle (internal capsule); $\mathrm{lg}=$ lingual gyrus; $\mathrm{m}=$ midbrain; $\mathrm{mb}=$ mammillary body; $\mathrm{mr}=$ marginal ramus of cingulate sulcus; $\mathrm{mt}=$ mammillothalamic tract; $\mathrm{oc}=$ optic chiasm; or = optic radiation; ot = optic tract; $\mathrm{pb}=$ pineal body; $\mathrm{pc}=$ precuneus; $\mathrm{pcl}=$ paracentral lobule; $\mathrm{pcs}=$ posterior calcarine sulcus; $\mathrm{pg}=$ paraterminal gyrus; pos $=$ parietooccipital sulcus; $\mathrm{ppc}=$ prepiriform cortex (tip of the parahippocampal gyrus); $\mathrm{prcu}=$ precuneus; $\mathrm{pt}=$ pulvinar thalami; $\mathrm{ptp}=$ posterior thalamic peduncle (internal capsule); $\mathrm{r}=$ rostrum of corpus callosum; rcc = radiations of corpus callosum; sa = subcallosal area; sas = sagittal stratum; sm = stria medullaris thalami; $\mathrm{sn}=$ substantia nigra; $\mathrm{sp}=$ splenium of corpus callosum; $\mathrm{ss}=$ subcallosal stratum; $\mathrm{st}=$ stria terminalis; stp = superior thalamic peduncle (internal capsule); $\mathrm{t}=$ thalamus; $\mathrm{ta}=$ tapetum of corpus callosum; $\mathrm{t} \mathrm{p}=$ temporal pole; $\mathrm{u}=$ uncus. 
callosum, the crus of the fornix and the hippocampal commissure are exposed. The fimbria can be traced to the crus, body, and column of the fornix, and terminates in the mammillary body. The frontal horn, body, and atrial portions of the lateral ventricle with the choroid plexus, as well as the head and body portions of the caudate nucleus are demonstrated (Fig. 1C).

The hippocampus, choroid plexus, fimbria, crus, and body portions of the fornix compose the entire anatomy of the lateral ventricle. After further removal of the medial frontoparietal cortex, removal of the cingulum and further dissection of the corpus callosum reveals the radiating fibers of the corpus callosum. The callosal fibers form a major portion of the commissural system and serve to interconnect the hemispheres. The genu portion of these fibers is known as the forceps minor, and the splenial portion is called the forceps major. The uncus is deflected to separate the amygdala from its complex connections, revealing the temporal horn of the lateral ventricle. The hippocampus is dissected free of the collateral eminence to gain entrance into the collateral sulcus. The tail of the hippocampus and the fornix are separated from the choroid plexus along the choroidal fissure. The fornix is incised at the junction of the body and column, and the choroid plexus is removed along the choroidal fissure. The stria terminalis, located between the caudate nucleus and thalamus, connects the bed nucleus of the stria terminalis and parts of the hypothalamus to the amygdala (Fig. 1D).

The removal of the frontal horn ependyma (which is a single layer of specialized epithelium lining the ventricles) and the body of the lateral ventricle allows exposure of the head and body of the caudate nucleus and the subcallosal stratum. The subcallosal stratum is a subependymal structure located between the caudate nucleus and the radiations of the corpus callosum. The caudate nucleus is observed to extend along the wall of the lateral ventricle and the tail of the caudate reaches forward to the level of the amygdala. The caudate nucleus has the same soft consistency as the putamen. Removal of the head and body portions of the caudate nucleus reveals the anterior and superior thalamic peduncles. The next step involves dissecting away the anterior portions of the subcallosal stratum and the radiations of the corpus callosum to allow identification of the extensions of the anterior and superior thalamic peduncles to the cortex. These peduncles are the anteromedial portion of the internal capsule, and they connect the frontoparietal regions of the cortex with the thalamus (Fig. 1E).

After total removal of the ependyma lining the lateral wall and roof of the lateral ventricle, the posterior portion of the subcallosal stratum and the tapetum of the corpus callosum are demonstrated, both of which were found to be subependymal structures. The tapetum, a subgroup of callosal fibers in the splenial region, forms the roof and lateral wall of the atrial portion of the lateral ventricle and sweeps around the temporal horn, thereby separating the fibers of the optic radiation from the temporal horn. Further removal of the caudate nucleus exposes the posterior and inferior thalamic peduncles. During our fiber dissection, identification of the precise location of the border separating the tapetum and the subcallosal stratum eluded us. Nevertheless, we did note a distinct difference between these two structures, and we suspect that the border lies between the body and the atrial portions of the lateral ventricle. In the subcallosal stratum, we were unable to identify a definite fiber system. There was, however, a fiber system clearly present in the tapetum. In addition, we made a significant observation; that the subcallosal stratum has fine, microscopic connections with the superior margin of the caudate nucleus (Fig. 1F).

The tapetum extends from the splenium, forming the lateral wall of the atrium and the roof of the temporal horn beneath the ependyma. After removing the remaining posterior layer of the subcallosal stratum, we dissected away the tapetum, the stria terminalis, and the amygdala, exposing the entire anatomy of the anterior, superior, posterior, and inferior thalamic peduncles as well as the optic radiation over the roof of the temporal horn. Resection of the body of the corpus callosum further exposed the superior thalamic peduncle. The thalamic peduncles as a whole form the medial portion of the internal capsule and connect the cerebral cortex to the thalamus. The inferior thalamic peduncle connects the temporal lobe to the thalamus. Following the column of the fornix into the hypothalamus demonstrates its connection with the mammillary body. The anterior commissure is located anterior to the column of the fornix. Further dissection into the hypothalamus and thalamus reveals the column of the fornix, optic tract, mammillary body, and mammillothalamic tract, which is also known as the tract of Vicq d'Azyr. Extensive dissection into the thalamus reveals its connections with the thalamic peduncles (Fig. 1G).

Removal of the anterior, superior, posterior (which includes the optic radiation), and inferior thalamic peduncles together with the thalamus and the lateral geniculate body concludes the dissection and demonstrates the whole corona radiata and the lateral portion of the internal capsule from a medial view, and the cerebral peduncle. These structures are composed of frontopontine fibers, pyramidal tract, and occipitopontine and temporopontine fibers (Fig. 1H).

\section{Surgical Considerations}

The striking advances in neurovisualization technology confirm the observations of neuropathologists, neurologists, and neurosurgeons that each type of CNS lesion has a predilection to present in distinct sites in osseous, meningeal, cisternal, parenchymal, ventricular, or vascular compartments. In each of these locations, the lesions may often reach a considerable size without causing any or only discreet signs and symptoms. It can be assumed that a lesion may compress and displace normal brain structures to a greater degree, but lack the capacity to transgress and destroy the unique architecture of the gray and white matter of the CNS. This fact affords us the opportunity to devise and initiate adequate treatment plans.

The main principle of a neurosurgical procedure is always to perform a pure lesionectomy, using tactics to avoid compromising normal homeostasis of the CNS. This surgical principle becomes a challenge to uphold when considering deep, localized, so-called "midline lesions," which may originate from the medial part of the frontal, parietal, or occipital lobe; from the anterior, middle, or posterior parts of the cingulate gyrus; from the parasplenial region (posterior cingulate gyrus, inferior precuneus, and posterior parahippocampal gyrus); corpus callosum; thalamus; hypothalamus; or third and lateral ventricles. All lesions in these locations (tumors, AVMs, and cavernomas) can be explored 
and removed through an anterior, middle, or posterior interhemispheric, and supracerebellar-infratentorial approach (Fig. 2). The specific method of these approaches prevents infliction of harm to the dorsal neopallial areas and to the connective fiber systems of the white matter.

It is an indisputable fact that falcine and callosal lesions, and saccular aneurysms of $\mathrm{A}_{2}$ and $\mathrm{A}_{3}$ segments are explored and removed (or occluded with clips) via an exploration into the interhemispheric fissure. This is considered a routine approach for the majority of midline lesions. Endovascular and gamma knife surgeries have proven effective for some types of tumors and for AVMs of smaller dimensions. Computer-assisted stereotactic, endoscopic, or microsurgical procedures are certainly accurate in targeting these lesions, but transcerebral trajectories to approach a lesion surgically are accompanied by unavoidable injuries to the neopallial cortices and connective fiber systems of the white matter.

\section{ILLUSTRATIVE CASES}

A few patients with typical deep midline lesions chosen from several hundred cases treated by the senior author (M.G.Y.) at the University of Arkansas for Medical Sciences in Little Rock illustrate the effectiveness of interhemispheric approaches (Figs. 3-12).

\section{Case 1}

This 26-year-old woman suffered from simple and complex partial seizures and impairment of her short-term memory. Admission MR images (Fig. 3A-C) demonstrated a large tumor occupying the anterior and subcallosal areas of the right cingulate gyrus. The tumor was removed via a frontal interhemispheric approach (Fig. 3D-F, postoperative MR images). Histological studies revealed a pilo-

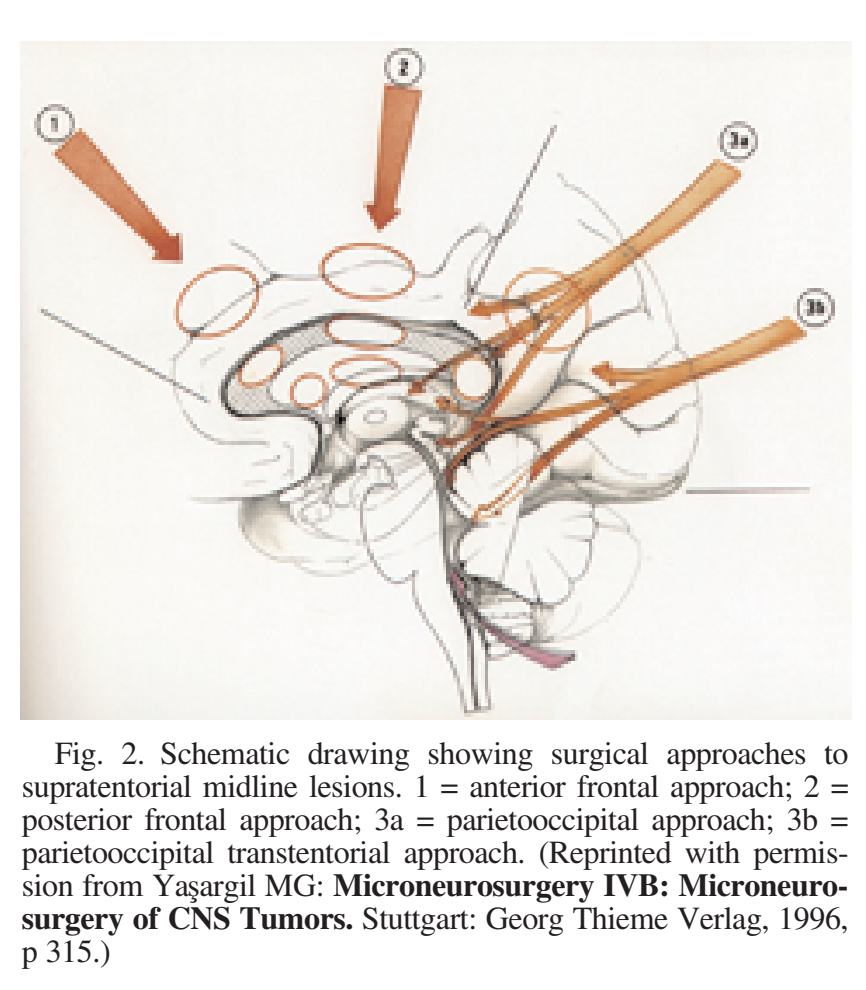

cytic astrocytoma. She had no neurological deficits pre- or postoperatively. Her postoperative course was uneventful. There was a remarkable improvement in her short-term memory difficulties, and she regained her full working capacity.

\section{Case 2}

This 10-year-old girl with chronic headaches underwent CT scanning, which demonstrated occlusive hydrocephalus due to a lesion in the third ventricle. After a ventriculoatrial shunt was placed, the lesion was explored and completely removed through a right frontal parasagittal osteoplastic craniotomy, via an anterior interhemispheric transcallosal-transforaminal approach. Histological studies revealed a pilocytic astrocytoma. The patient had no neurological, mental, or endocrine deficits pre- or postoperatively. Nine years of follow up revealed no recurrence of the tumor (Fig. $4 \mathrm{~A}-\mathrm{C}$, preoperative and Fig. 4D-F, postoperative MR images).

\section{Case 3}

This 12-year-old boy presented with headache after hitting a soccer ball with his head. The admission CT and MR imaging studies revealed a well-defined lesion in the right anterior lateral thalamic region, which was removed via an interhemispheric transcallosal approach. Histological stud-

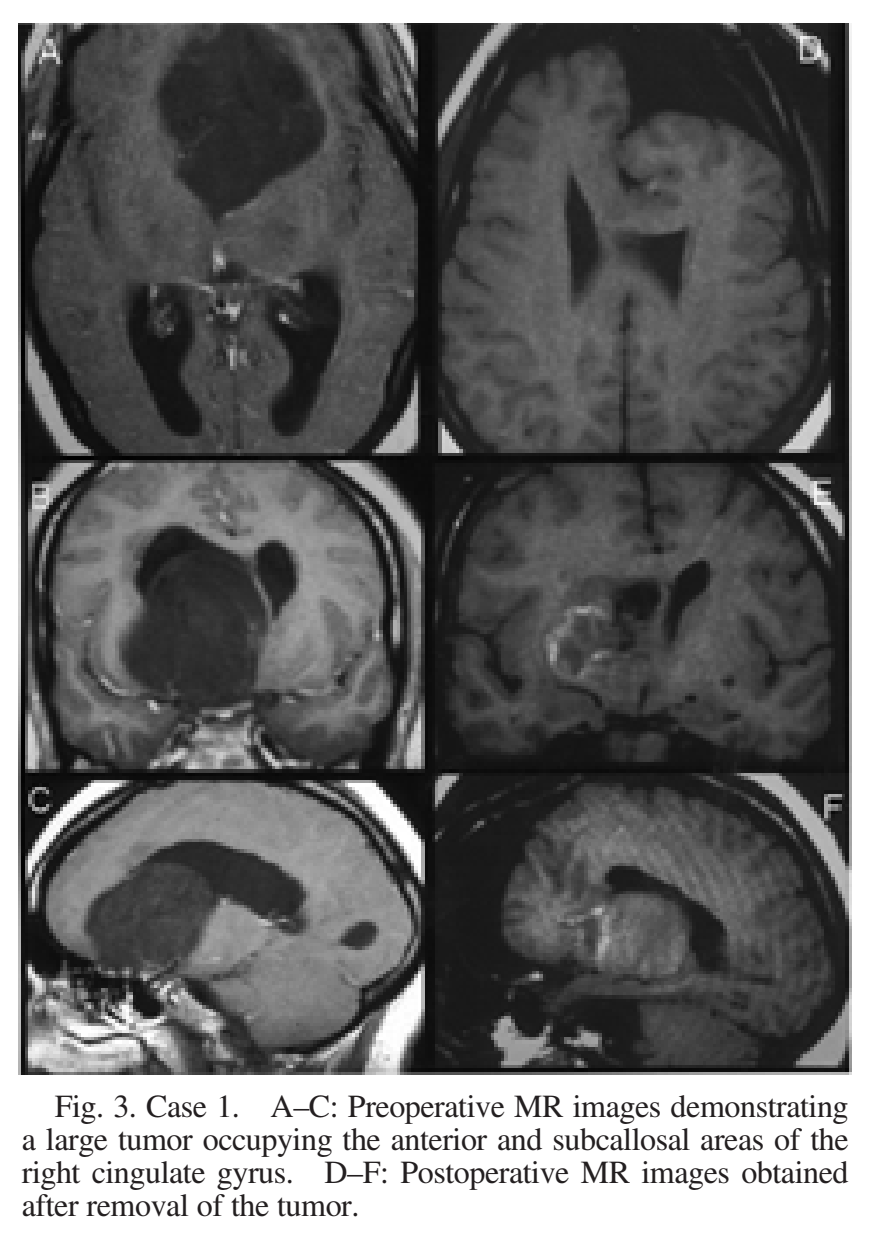


ies revealed pilocytic astrocytoma. The patient's pre- and postoperative neurological and mental status was normal (Fig. 5A-C, preoperative and Fig. 5D-F, postoperative MR images).

\section{Case 4}

This 7-year-old girl experienced progressive weakness distally in her right leg. The admission CT and MR imaging studies revealed a large tumor originating in the region of the septum pellucidum, extending through the middle of the corpus callosum and interhemispheric fissure to the surface of the left pre- and postcentral gyri. The lesion was explored and completely removed through a left posterior frontal parasagittal craniotomy, and further exploration was performed along the interhemispheric fissure in a superoinferior dissection (Fig. 6A and B, preoperative and Fig. $6 \mathrm{C}$ and $\mathrm{D}$, postoperative MR images). The postoperative course was uneventful, and the foot process recovered fully within a few months. Histological findings remained inconclusive; the tumor was thought to be either a neurocytoma or an oligodendroglioma.

\section{Case 5}

This 42-year-old woman suffered single and complex partial seizures. The admission CT and MR imaging studies demonstrated a well-circumscribed lesion within the right precuneus area, with extension into the posterior part of the right parahippocampal gyrus. With the patient in a sitting position, the lesion was explored and completely removed through a right parietooccipital osteoplastic craniotomy and via a posterior interhemispheric approach (Fig. 7A-C, preoperative and Fig. 7D-F, postoperative MR images). The histological studies revealed low-grade oligodendroglioma. The patient was free of neurological and mental deficits pre- and postoperatively, and her visual field is normal. She has regained her full working capacity and has had no seizures.

\section{Case 6}

This 36-year-old woman reported difficulty with reading and memory problems. She had right hemianopia and papilledema. The admission MR images (Fig. 8A-C) revealed a large lesion within the left precuneus and posterior parahippocampal gyrus. The dorsal part of the tumor had been removed in another hospital. The tumor was completely removed after a second exploration; this was done through a left parietooccipital craniotomy and via an interhemispheric approach with the patient in a sitting position (Fig. 8D-F, postoperative MR images). Histological studies revealed a low-grade oligodendroglioma. There were no neurological and mental deficits and no visual field deficits pre- or postoperatively. The patient regained her full working capacity, and has had no seizures.

\section{Case 7}

This 25-year-old woman suffered from a headache that increased in intensity over 5 months, fatigue, and shortterm memory problems. The admission CT and MR imaging studies demonstrated a compact lesion in the left atrium. With the patient in a sitting position, the tumor was explored through a left parietooccipital osteoplastic cran- iotomy, via a posterior interhemispheric approach. The dorsomedial extension of the tumor into the posterior part of cingulate gyrus was identified. Through a 10-mm-long incision, the well-encapsulated, very vascularized lesion was completely removed. Histological studies revealed an atypical meningioma. The patient's pre- and postoperative neurological and mental status were found to be normal; in particular no visual field deficit was detected. She regained her full working capacity (Fig. 9A-C, preoperative and Fig. 9D-F, postoperative MR images).

\section{Case 8}

In this 42-year-old woman, who had suffered from typical temporal seizures for a couple of years, admission MR imaging studies (Fig. 10A-C) revealed a large, left-sided mediobasal tumor extending into the dorsolateral region of the mesencephalon. The tumor was explored and removed (except for the amygdala area) via a supracerebellar-transtentorial approach (Fig. 10D-F, postoperative MR images). The patient's postoperative course was uneventful. Preoperatively and postoperatively she had no neurological or mental deficits, and her visual field was intact. The histological studies revealed a low-grade oligodendroglioma. After surgery, the seizures did not recur and the patient could continue to work at her full capacity.

\section{Case 9}

In this 12-year-old girl suffering from a progressive right hemisyndrome but no visual field defect, the admission MR imaging studies revealed a well-circumscribed intrinsic tumor in the left posterior thalamic region (Fig. 11A-C). With the patient in a sitting position, the lesion was explored via a left supracerebellar-transtentorial approach, and an anaplastic astrocytoma was radically removed with the aid of an operating microscope (Fig. 11D-F, postoperative MR images). Postoperatively there was rapid improvement of the hemisyndrome, and there was no visual field deficit. The adjuvant radio- and chemotherapy could not change the course of her disease, and she died 2 years postsurgery.

\section{Case 10}

This 45-year-old woman suffered an onset of a right hemisyndrome and homonymous hemianopia. The admission MR images (Fig. 12A-C) demonstrated a cavernoma in the left posterior thalamic region. With the patient in a sitting position, this lesion was explored via a supracerebellar-transtentorial approach, and we were able to remove the lesion completely (Fig. 12D-F). The histological findings confirmed a cavernoma. Postoperatively the moderate hemiparesis improved remarkably, whereas the visual field deficit remained unchanged.

\section{DISCUSSION}

The planning of a neurosurgical procedure incorporates a study of the parenchymal, vascular, cisternal, and ventricular architecture of the brain on MR imaging, MR angiography, MR venography, and serial cerebral angiograms, which are usually analyzed in the axial (base-up > topdown), coronal (anteroposterior $>$ posteroanterior), and 
M. G. Yaşargil, U. Türe, and D. C. H. Yaşargil

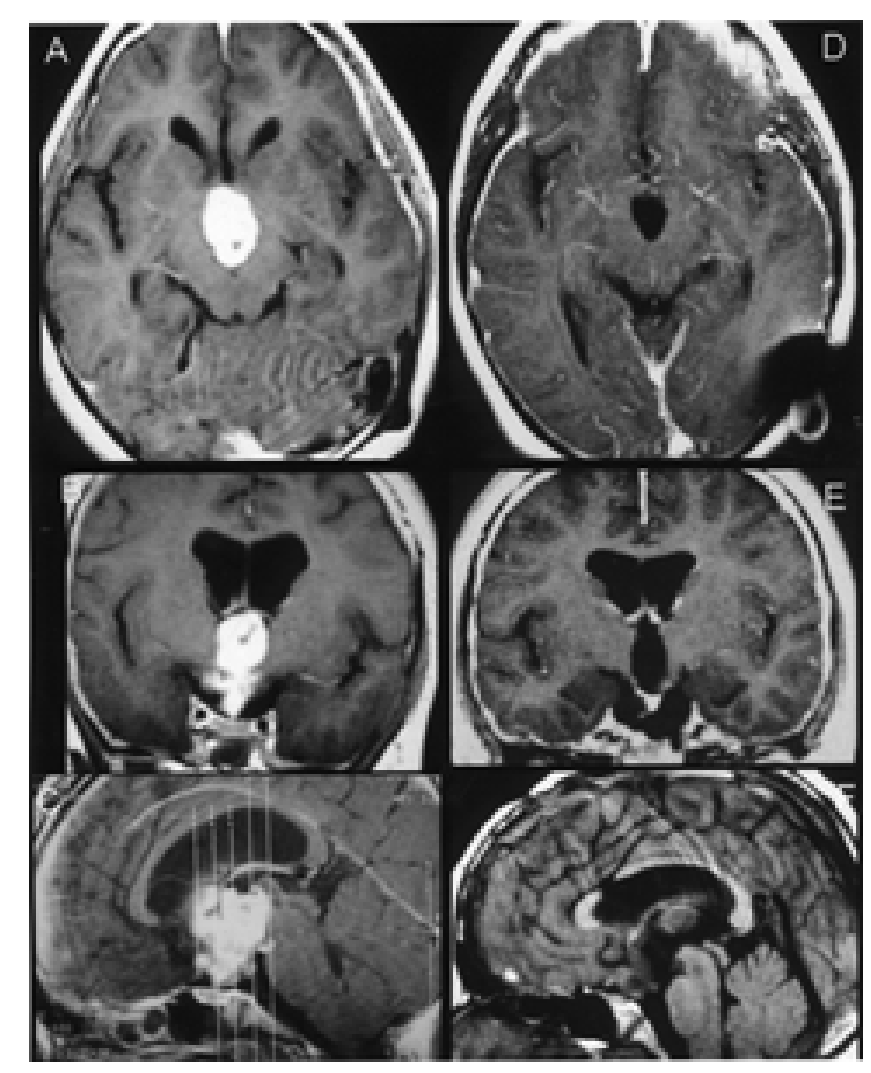

Fig. 4. Case 2. A-C: Preoperative MR images demonstrating occlusive hydrocephalus due to a lesion in the third ventricle. D-F: Postoperative MR images obtained after complete removal of the lesion.

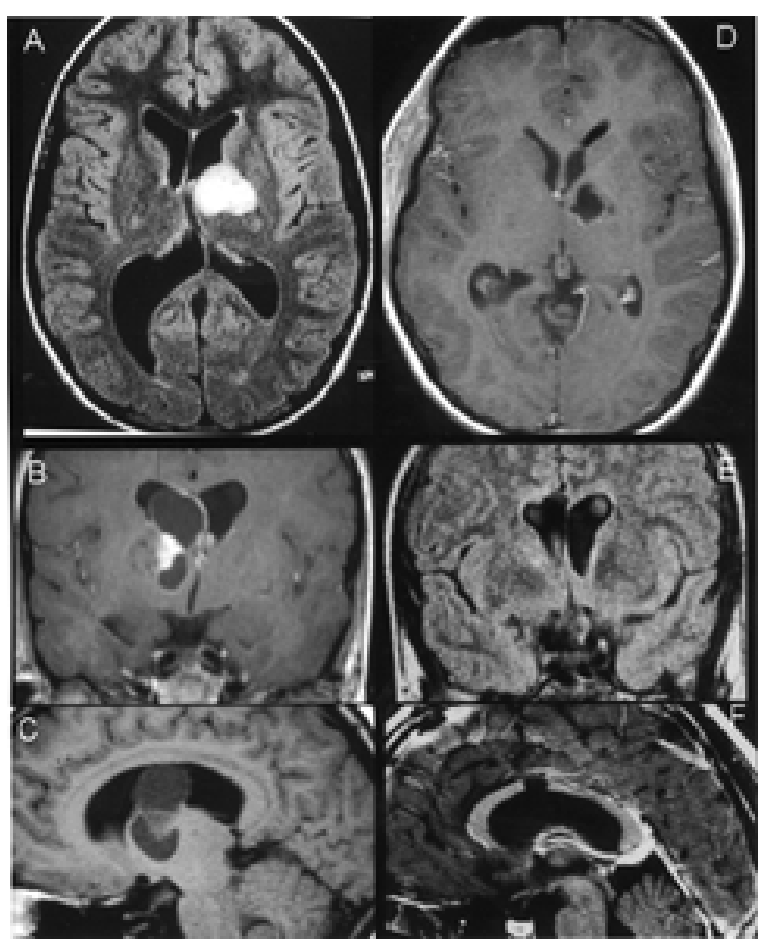

Fig. 5. Case 3. A-C: Preoperative MR images revealing a well-defined lesion in the right anterior lateral thalamic region. D-F: Postoperative MR images obtained after the lesion was removed.

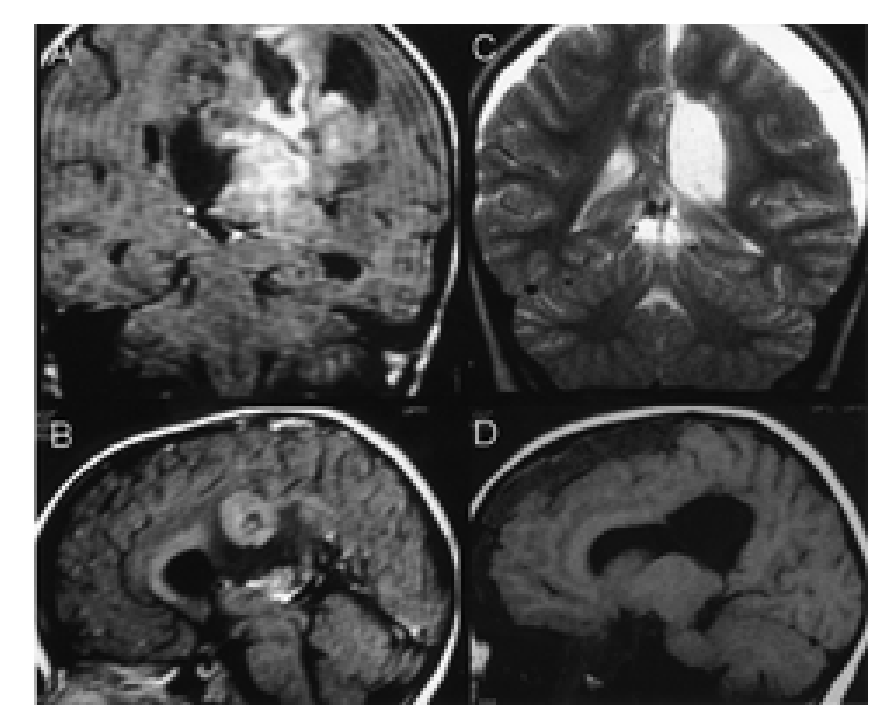

Fig. 6. Case 4. A and B: Preoperative MR images revealing a large tumor originating in the region of the septum pellucidum and extending through the middle of the corpus callosum and interhemispheric fissure to the surface of the left pre- and postcentral gyri. C and D: Postoperative MR images obtained after exploration and complete removal of the tumor.

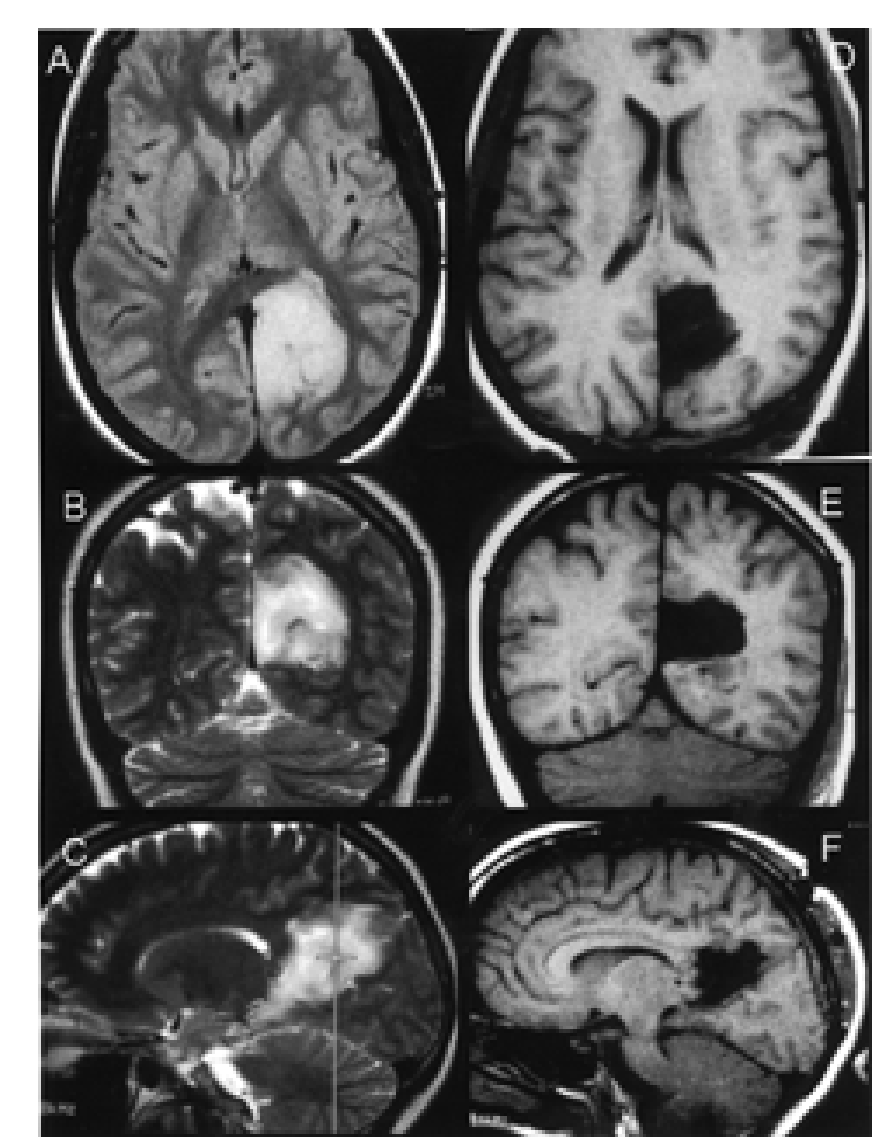

Fig. 7. Case 5. A-C: Preoperative MR images demonstrating a well-circumscribed lesion within the right precuneus area, with extension into the posterior part of the right parahippocampal gyrus. D-F: Postoperative MR images obtained after exploration and total resection. 


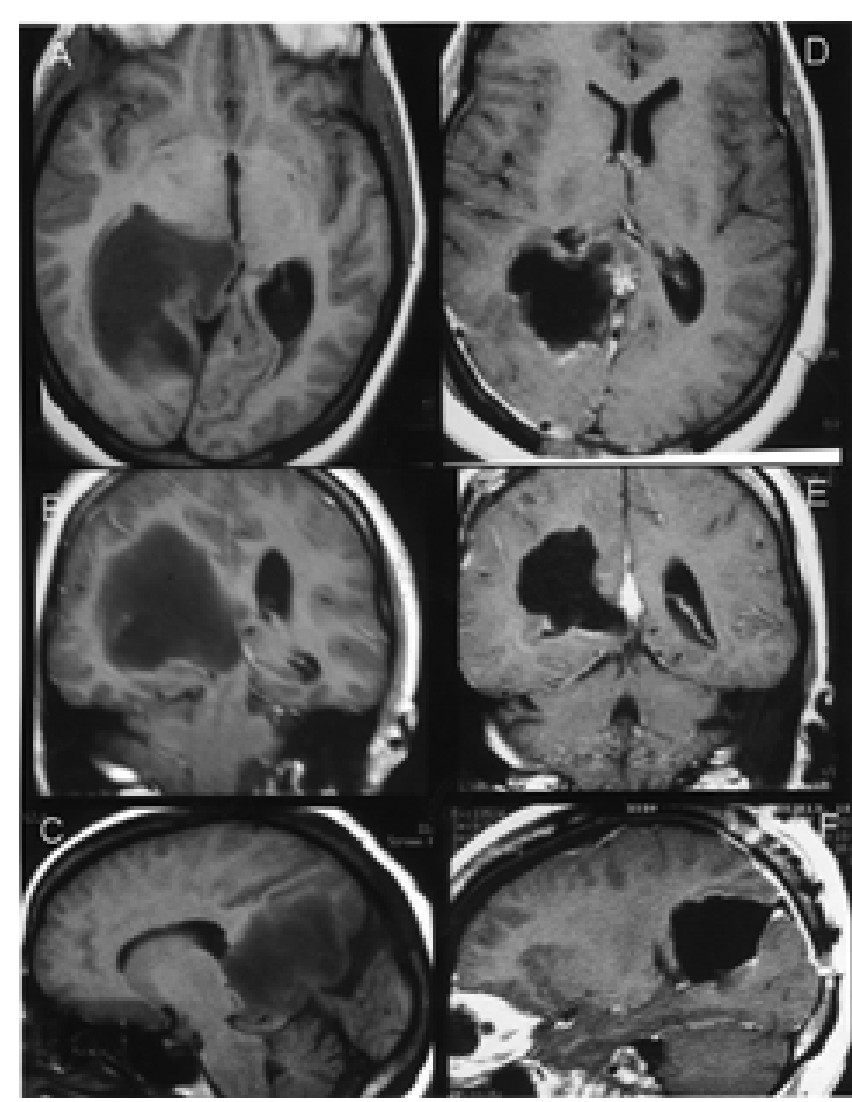

Fig. 8. Case 6. A-C: Preoperative MR images revealing a large lesion within the left precuneus and posterior parahippocampal gyrus. D-F: Postoperative MR images obtained after exploration and complete resection of the lesion.

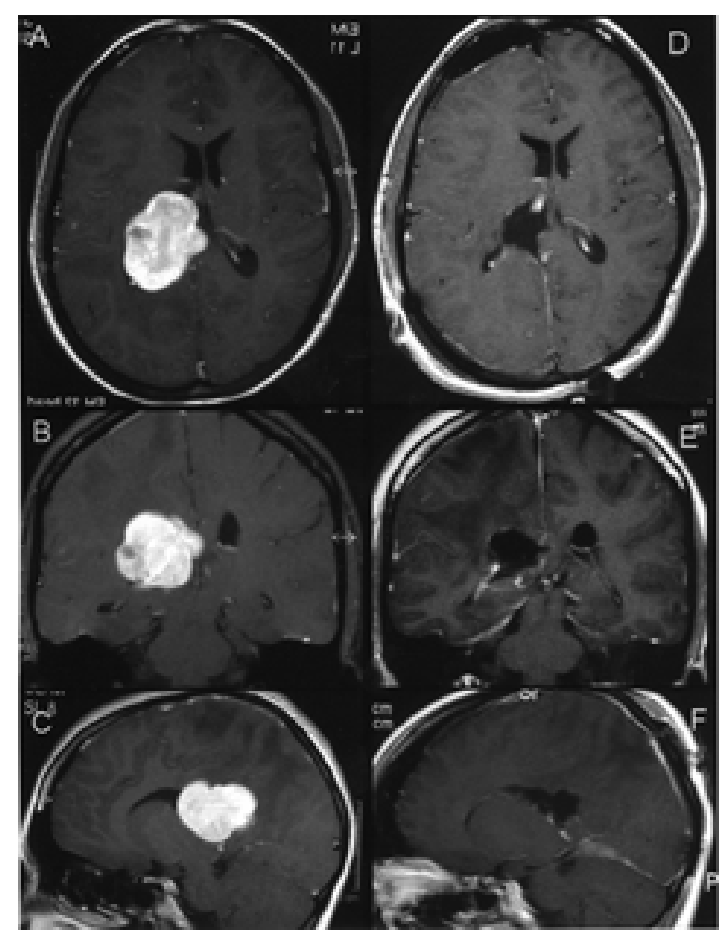

Fig. 9. Case 7. A-C: Preoperative MR images demonstrating a compact lesion in the left atrium. D-F: Postoperative MR images obtained after complete removal of the tumor.

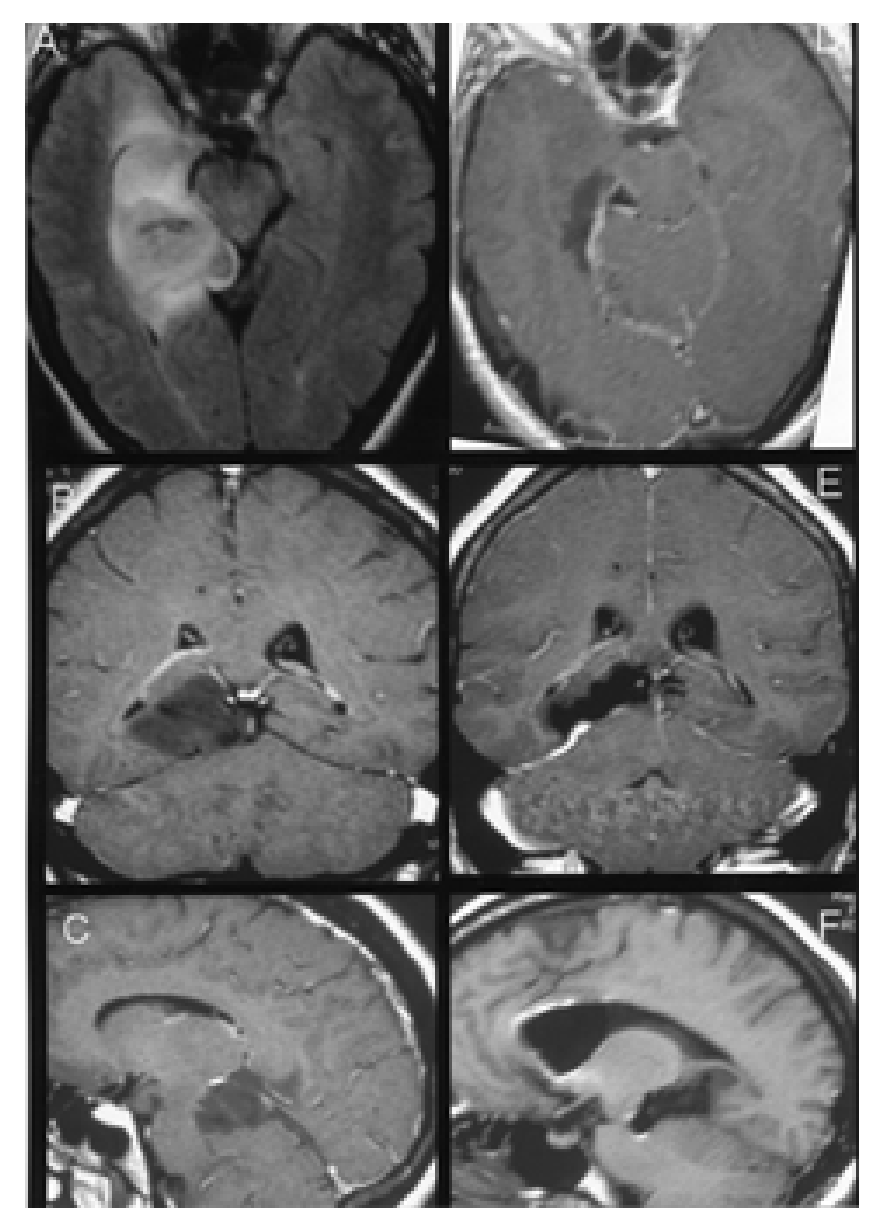

Fig. 10. Case 8. A-C: Preoperative MR images revealing a large, left-sided mediobasal tumor extending into the dorsolateral region of the mesencephalon. D-F: Postoperative MR images obtained after tumor removal.

sagittal view (lateromedial $>$ mediolateral). Because the lesions are explored with the patient either supine or in a sitting position, but are approached via the interhemispheric fissure in a mediolateral direction, the related fiber system of the white matter is also shown in a mediolateral direction in the dissected cadaveric brains in this study.

Inferiorly extending tumors of the hypothalamus and third ventricle can be explored and resected via a pterional-transsylvian and translamina-terminalis approach. Tumors in the posterior part of the third ventricle and the thalamus are approached via the posterior interhemispheric fissure or via a suboccipital-supracerebellar route.

Lesions in the third ventricle and the anterior two thirds of the lateral ventricle are explored through the anterior or middle part of the interhemispheric fissure, and lesions in the trigonum (atrium) are explored via a posterior interhemispheric approach with the patient in the sitting position. Exploration of the ventricle requires a small incision $(10-15 \mathrm{~cm})$ in the commissural fiber system of the corpus callosum. ${ }^{22-25}$ One exception to this recommendation is when a tumor epands to the surface of the frontal or parietal lobe. This, however, is an extremely rare occurrence.

Dorsal transcerebral approaches traverse neocortical areas, and injuries to cortices and to the complex stratification 


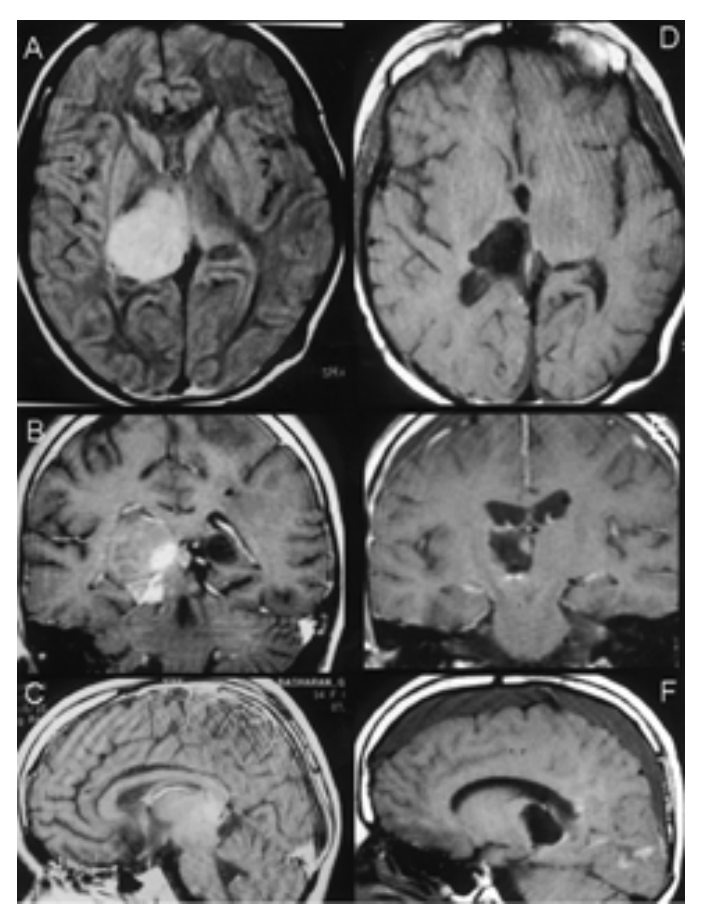

Fig. 11. Case 9. A-C: Preoperative MR images revealing a well-circumscribed intrinsic tumor in the left posterior thalamic region. D-F: Postoperative MR images obtained after radical tumor removal.

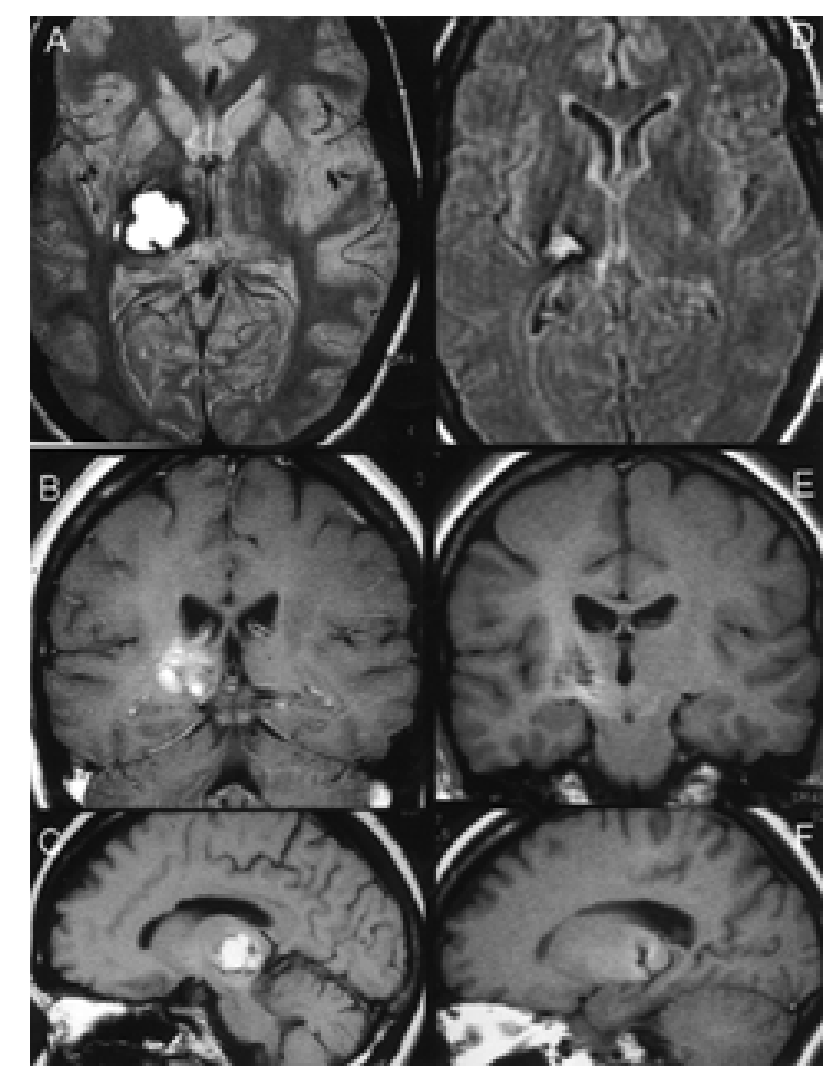

Fig. 12. Case 10. A-C: Preoperative MR images demonstrating a cavernoma in the left posterior thalamic region. D-F: Postoperative MR images obtained after complete removal of the lesion. of associative, commissural, and projection fiber systems are impossible to avoid. Interhemispheric transcallosal approaches are definitely the preferred surgical strategies, offering good access to the lesion, permitting conservation of normal tissue and structures, and resulting in a positive outcome for the patient.

\section{References}

1. Apuzzo MLJ, Litofsky NS: Surgery in and around the anterior third ventricle, in Apuzzo MLJ (ed): Brain Surgery: Complication Avoidance and Management. New York: Churchill-Livingstone, 1993, pp 541-580

2. Bellotti C, Pappada G, Sani R, et al: The transcallosal approach for lesions affecting the lateral and third ventricles. Surgical considerations and results in a series of 42 cases. Acta Neurochir 111:103-107, 1991

3. D'Angelo VA, Galarza M, Catapano D, et al: Lateral ventricle tumors: surgical strategies according to tumor origin and development - a series of 72 cases. Neurosurgery 56 (Suppl 1): 36-45, 2005

4. Dandy WE: Benign Tumors in the Third Ventricle of the Brain: Diagnosis and Treatment. Springfield, IL: Charles C Thomas, 1933

5. Geffen G, Walsh A, Simpson D, et al: Comparison of the effects of transcortical and transcallosal removal of intraventricular tumours. Brain 103:773-788, 1980

6. Hütter BO, Spetzger U, Bertalanffy $\mathrm{H}$, et al: Cognition and quality of life in patients after transcallosal microsurgery for midline tumors. J Neurosurg Sci 41:123-129, 1997

7. Jeeves MA, Simpson DA, Geffen G: Functional consequences of the transcallosal removal of intraventricular tumours. J Neurol Neurosurg Psychiatry 42:134-142, 1979

8. Klingler J: Erleichterung der makroskopischen Praeparation des Gehirns durch den Gefrierprozess. Schweiz Arch Neurol Psychiat 36:247-256, 1935

9. Konovalov AN, Gorelyshev SK, Khuhlaeva EA: Surgery of diencephalic and brainstem tumors, in Schmidek HH, Sweet WH (eds): Operative Neurosurgical Technique. Indications, Methods and Results, ed 3. Philadelphia: Saunders, Vol 1, 1995, pp 765-782

10. Ludwig E, Klingler J: Atlas cerebri humani der innere Bau des Gehirns dargestellt auf Grund makroskopischer Präparate. Basel: Karger S, 1956

11. Misra BK, Rout D, Padamadan J, et al: Transcallosal approach to anterior and mid-third ventricular tumors-a review of 62 cases. Ann Acad Med Singapore 22 (Suppl 3):435-440, 1993

12. Pendl G: Pineal and Midbrain Lesions. Wien: Springer, 1985

13. Rhoton AL Jr: The lateral and third ventricles. Neurosurgery 51 (Suppl 4):S207-S271, 2002

14. Rosenfeld JV, Harvey AS, Wrennall J, et al: Transcallosal resection of hypothalamic hamartomas, with control of seizures, in children with gelastic epilepsy. Neurosurgery 48:108-118, 2001

15. Standefer M, Bay JW, Trusso R: The sitting position in neurosurgery: a retrospective analysis of 488 cases. Neurosurgery 14:649-658, 1984

16. Timurkaynak E, Rhoton AL Jr, Barry M: Microsurgical anatomy and operative approaches to the lateral ventricles. Neurosurgery 19:685-723, 1986

17. Türe U, Yaşargil MG, Al-Mefty O: The transcallosal-transforaminal approach to the third ventricle with regard to the venous variations in this region. J Neurosurg 87:706-715, 1997

18. Türe U, Yaşargil MG, Friedman AH, et al: Fiber dissection technique: lateral aspect of the brain. Neurosurgery 47:417-427, 2000

19. Türe U, Yaşargil MG, Pait TG: Is there a superior occipitofrontal fasciculus? A microsurgical anatomic study. Neurosurgery 40:1226-1232, 1997 
20. Voigt K, Yaşargil MG: Cerebral cavernous hemangioma or cavernomas. Incidence, pathology, localization, diagnosis, clinical features and treatment. Review of the literature and report of an unusual case. Neurochir 19:59-68, 1976

21. Woiciechowsky C, Vogel S, Lehmann R, et al: Transcallosal removal of lesions affecting the third ventricle: an anatomic and clinical study. Neurosurgery 36:117-123, 1995

22. Yaşargil MG: Microneurosurgery I: Microsurgical Anatomy of the Basal Cisterns and Vessels of the Brain, Diagnostic Studies, General Operative Techniques and Pathological Considerations of the Intracranial Aneurysms. Stuttgart: Georg Thieme Verlag, 1984, pp 5-168

23. Yaşargil MG: Microneurosurgery IIIB: Arm of the Brain, Clinical Considerations, General and Special Operative Techniques, Surgical Results, Nonoperated Cases, Cavernous and Venous Angiomas, Neuroanesthesia. Stuttgart: Georg Thieme Verlag, 1988

24. Yaşargil MG: Microneurosurgery IVB: Microneurosurgery of CNS Tumors. Stuttgart: Georg Thieme Verlag, 1996, pp 237-342
25. Yaşargil MG, Türe U, Roth P: Combined approaches, in Apuzzo MLJ (ed): Surgery of the Third Ventricle, ed 2. Baltimore: Williams \& Wilkins, 1998, pp 541-552

26. Yamamoto I, Rhoton AL Jr, Peace DA: Microsurgery of the third ventricle: Part I. Microsurgical anatomy. Neurosurgery 8:334-356, 1981

27. Yonekawa Y, Imhof HG, Taub E, Curcic M, Kaku Y, Roth P, Wieser HG, Groscurth P. Supracerebellar transtentorial approach to posterior temporomedial structure. J Neurosurg 94:339-345, 2001

Manuscript received April 25, 2005.

Accepted in final form May 11, 2005.

Address reprint requests to: M. Gazi Yaşargil, M.D., Department of Neurosurgery, University of Arkansas for Medical Sciences, 4301 West Markham, \#507, Little Rock, Arkansas 72205. 